\title{
Manufacture and Delivery Scheduling for Multiple Customers on a Single Machine with Availability Constraint
}

\author{
Jing Fan \\ College of Arts and Science, \\ Shanghai Polytechnic University \\ Shanghai, China. \\ fanjing@sspu.edu.cn
}

\begin{abstract}
In this paper we consider a scheduling problem with the joint consideration of manufacture and delivery for multiple customers. Since the machine is not always available due to breakdown or maintenance during the manufacture period, the interrupted job is resumable after the machine becomes available again. In the delivery period, amounts of vehicles with fixed load capacities deliver completed jobs in batches to $K$ customers. Only jobs belonging to the same customer can be delivered together in the same shipment. The cost of each delivery for different customer is different. Our goal is to minimize the sum of total departure time and total delivery cost. The problem is solved optimally by a dynamic programming algorithm with polynomial time $O\left(n^{K+2}\right)$, where $n$ is the number of jobs. The performance of an instance is shown the effectiveness of the algorithm.
\end{abstract}

Keywords-Manufacture; Delivery; Scheduling; Multiple Customers; Dynamic Programming Algorithm

\section{INTRODUCTION}

\section{A. Background of the problem}

In the real industrial settings, each job should go through two stages of manufacture arrangement and product delivery. In the manufacture period, there exists one machine to process jobs. It is noted that the machine may become unavailable due to breakdown or maintenance during the manufacturing period. The jobs may be interrupted by the unavailable interval and may be resumable after the machine turns into available again. In the delivery period, homogeneous vehicles with fixed load capacities deliver jobs to the customer. A completed job is permitted waiting until some other jobs of the same customer are finished processing. Then they compose one delivery batch, that is to say, these jobs are delivered as one batch. In fact, the transportation task is always performed by the third-party logistics. The cost of each transportation task or delivery for the different customer is different but fixed.

If there is only one job in each batch, the customer is satisfied with the speed of delivery while the manufacturer must pay the highest wages for the third-party logistics. If there are all jobs of the same customer in one batch, the manufacturer must pay the least wages for the third-party logistics but the customer, which is the last one received completed jobs, ought to complain the production ability of the manufacturer. Hence, the manager of the manufacturer must consider not only how to transport products to the customers as fast as possible, but also how to decrease the expenses on delivery as little as they could.

\section{B. Description of the problem}

The problem we study can be described as follows. Given a set of n jobs $J=\left\{J_{1}^{1}, J_{2}^{1}, \ldots, J_{n_{1}}^{1}, J_{1}^{2}, J_{2}^{2}, \ldots, J_{n_{2}}^{2}, \ldots, J_{1}^{K}, J_{2}^{K}, \ldots, J_{n_{K}}^{K}\right\}$,

in which jobs $J_{1}^{i}, J_{2}^{i}, \ldots, J_{n_{i}}^{i}$ belong to the $i$-th customer for $i=1,2, \ldots, K$. Job $J_{j}^{i}$ is available at time 0 to be processed on a single machine and then delivered to the $i$-th customer. The processing time of job $J_{j}^{i}$ is $p_{j}^{i}$ and its completion time is denoted by $C_{j}^{i}$. The machine $M$ is unavailable within a given time interval $I=[S, E]$, where $S<E$. There exist homogeneous available vehicles to deliver completed jobs, each of which can carry at most $c$ jobs in one batch. Only those completed jobs that belong to the same customer can be delivered together as one batch by one of the vehicles to that customer. The cost of each delivery batch for the $i$-th customer is denoted by $\lambda_{i}(i=1,2, \ldots, K)$, and the total delivery cost is denoted by $T$. The departure time of job $J_{j}^{i}$, denoted by $D_{j}^{i}$, is defined as the time by which it is departed to the customer from the machine $M$. The objective is to minimize the sum of total departure time and total delivery cost of all jobs.

By the notation of Chen [1], the problem is represented by

(P): $1|r-a| V(\infty, c)$, direct $|K| \Sigma \Sigma D_{j}^{i}+T$,

where ' $r$-a' denote the job is resumable, ' $V(\infty, c)$ ' describes the situation of vehicles, in which ' $\infty$ ' represents the number of vehicle is unlimited and ' $c$ ' is the load capacity, and 'direct' denotes that only jobs belonging to the same customer can be delivered together in the same shipment, and ' $\Sigma \Sigma D_{j}^{i}+T$ ' is the objective function. 


\section{REVIEW OF RELATED WORK}

In the most literatures of scheduling problem concerned with integration of manufacture and delivery, we always assume that machines are continuously available for processing throughout the manufacture period. If there is one machine and homogeneous vehicles, Chen and Vairaktarakis [2] present an $O(n \log n+n c)$ dynamic programming algorithm, where $c$ is the capacity of the vehicle. If there exits only one vehicle without the capacity constraint, Hall and Potts [3] propose an $O\left(n^{4}\right)$ dynamic programming algorithm. Li et al. [4] provide an $O\left(n^{2}\right)$ dynamic programming algorithm subjected to the capacity constraint of vehicles.

Igor et al. study the on-line problems with one customer and multiple customers in 2007 [5] and in 2010 [6], respectively. Moreover, Chen has provided a survey on supply chain scheduling and presented a model representation scheme with five-field notation: $\alpha|\beta| \pi|\delta| \gamma$, where $\alpha, \beta, \pi, \delta$ and $\gamma$ represent the machine configuration, constraints of jobs, the delivery process, the number of customers and the objective function, respectively.

From 1990's, many literatures on the models with machine availability constraints sprang up. In the case of an unavailability interval, Lee [7] shows the SPT sequence can solve the resumable problem optimally to minimize the total completion times of jobs. And more reviews can be referred to Ying Ma et al [8]

It is notable that the literature associated manufacture and delivery scheduling with unavailability constraint is rare. Wang and Cheng [9] study the objective of minimizing the arrival time of the last delivery batch and the total delivery cost when there is only one capacitated vehicle to deliver jobs within a fixed time. If the jobs are resumable and need to be processed on a single machine, they provide an optimal polynomial time algorithm. Fan et al. [10] solve optimally the integrated scheduling of manufacture and delivery for one customer on a single machine with availability constraint by a dynamic programming algorithm if the jobs are resumable and the vehicles are homogeneous and without capacity limits.

To the best of our knowledge, the manufacture and delivery scheduling problem for multiple customers on a single machine with availability constraint has not been studied yet.

\section{SOLVING PROBLEM (P)}

In this section, we firstly analyze the optimal properties of the problem (P): $1|r-a| V(\infty, c)$, direct $|K| \Sigma \Sigma D_{j}^{i}+T$, then we present an optimal dynamic programming algorithm for it.

\section{A. Optimal Properties}

Now we analyze some properties of the optimal schedule to the problem $(\mathrm{P})$.

\section{Lemma}

For the problem $(\mathrm{P})$ :

$$
1|r-a| V(\infty, c), \text { direct }|K| \Sigma \Sigma D_{j}^{i}+T,
$$

there exists an optimal schedule that satisfies the following conditions:

(1) There is no idle time on the machine $M$ before all jobs are completed except the unavailable interval;

(2) In the manufacture period, jobs of each customer are processed in the shortest processing time first (SPT) order on the machine;

(3) In the delivery period, the departure time of a batch of jobs belonging to $i$-th customer is the completion time of the last completed job in that batch.

\section{Proof}

(1) If there is one idle time on the machine $M$ before the job $J_{j}^{i}$ is completed, we can move up the beginning time of the remain part of the job $J_{j}^{i}$ to fill the idle time, and the schedule of all unprocessed jobs move up the same time.

If there is one idle time on the machine $M$ after the job $J_{j}^{i}$ is completed, we can move up the remain jobs to fill the idle time.

Thus, the objective function value cannot increase.

(2) Let $\sigma$ be an optimal schedule for the problem (P). We will prove the conclusion by contradiction.

Suppose that in $\sigma^{*}$ job $J_{j}^{i}$ is immediately followed by $J_{k}^{i}$ and $p_{j}^{i}>p_{k}^{i}$ in the manufacture period. Then we can interchange job $J_{j}^{i}$ and job $J_{k}^{i}$ to obtain a new schedule, denoted by $\sigma^{\prime}$. And the delivery schedule of all jobs remains the same except for exchanging the deliveries of job $J_{j}^{i}$ and job $J_{k}^{i}$ in $\sigma^{\prime}$.

Denote the completion times of job $J_{j}^{i}$ and job $J_{k}^{i}$ in $\sigma^{*}$ by $C_{j}^{i}\left(\sigma^{*}\right)$ and $C_{k}^{i}\left(\sigma^{*}\right)$, respectively, and the completion times of job $J_{j}^{i}$ and job $J_{k}^{i}$ in $\sigma^{\prime}$ by $C_{j}^{i}\left(\sigma^{\prime}\right)$ and $C_{k}^{i}\left(\sigma^{\prime}\right)$, respectively. Thus, we have $C_{j}^{i}\left(\sigma^{*}\right) \geq C_{k}^{i}\left(\sigma^{\prime}\right)$ and $C_{k}^{i}\left(\sigma^{*}\right)=C_{j}^{i}\left(\sigma^{\prime}\right)$. Moreover, $D_{j}^{i}\left(\sigma^{*}\right) \geq D_{k}^{i}\left(\sigma^{\prime}\right), D_{k}^{i}\left(\sigma^{*}\right)=D_{j}^{i}\left(\sigma^{\prime}\right)$ and the departure time of other jobs does not change. Therefore, the objective function value of schedule $\sigma^{\prime}$ is not more than that of schedule $\sigma^{*}$.

This is in contradiction with the statement of $\sigma^{*}$ that $\sigma^{*}$ is an optimal schedule for the problem $(\mathrm{P})$.

(3) If the manufacture produce keeps the same and the departure time of a batch of jobs belonging to $i$-th customer is later than the completion time of the last completed job in that batch in the delivery period, we can deliver that batch of jobs of $i$-th customer at the completion time of the last completed job. The objective function value will be better than ever. Therefore, the departure time of a batch of jobs must be the completion time of the last completed job of that batch.

To obtain the optimal schedule of the problem $(\mathrm{P})$, we should sequence jobs of each customer in the SPT order according to (2) of Lemma, so that $p_{1}^{i} \leq p_{2}^{i} \leq \ldots \leq p_{n_{i}}^{i}$ for $i=1,2, \ldots, K$. 


\section{B. Optimal Dynamic Programming Algorithm}

In the dynamic programming algorithm, we introduce $\mathrm{K} 0$ 1 variables as follows:

$$
\theta_{l_{i}}=\left\{\begin{array}{ll}
1, & l_{i}>0 \\
0, & l_{i}=0
\end{array}(i=1,2, \cdots, K) .\right.
$$

If $l_{i}>0$, then $\theta_{l_{i}}=1$, which means that there are $l_{i}$ jobs of $i$-th customer in the current batch. Otherwise, $\theta_{l_{i}}=0$, which means that there are no jobs of $i$-th customer in the current batch.

Now we present the dynamic programming algorithm DP including $2 K$ dummy jobs

$$
J_{0}^{1}, J_{0}^{2}, \ldots, J_{0}^{K}, J_{N+1}^{1}, J_{N+1}^{2}, \ldots, J_{N+1}^{K}
$$

with $\quad p_{0}^{1}=p_{0}^{2}=\ldots=p_{0}^{K}=0$ and $p_{n_{1}+1}^{1}=p_{n_{2}+1}^{2}=\ldots$ $=p_{n_{K}+1}^{K}=0$.

\section{Algorithm DP}

Define $F\left(j_{1}, j_{2}, \ldots, j_{K}\right)$ as the minimum objective function value for jobs $J_{j_{1}}^{1}, J_{j_{1}+1}^{1}, \ldots, J_{n_{1}}^{1}$ of 1 st customer, $J_{j_{2}}^{2}, J_{j_{2}+1}^{2}, \ldots, J_{n_{2}}^{2}$ of 2 nd customer, $\ldots$, and $J_{j_{K}}^{K}, J_{j_{K}+1}^{K}, \ldots, J_{n_{K}}^{K}$ of $K$-th customer.

The initial condition:

$$
F\left(n_{1}+1, n_{2}+1, \ldots, n_{K}+1\right)=0 \text {; }
$$

The recurrence relation:

\begin{tabular}{|c|c|c|c|}
\hline Job $J_{j}^{i}$ & 1 & 2 & 3 \\
\hline Processing Time $p_{j}^{i}$ & 1 & 2 & 2 \\
\hline Customer $i$ & 1 & 1 & 2 \\
\hline
\end{tabular}

For $1 \leq j_{k} \leq n_{k}+1(k=1,2, \ldots, K)$,

$$
\begin{aligned}
& F\left(j_{1}, j_{2}, \ldots, j_{K}\right) \\
& =\min \{ \\
& \min _{1 \leq l_{1} \leq \min \left\{n_{1}-j_{1}+1, c\right\}}\left\{\theta_{l_{1}} l_{1} C\left(j_{1}+l_{1}-1, j_{2}-1, \ldots, j_{K}-1\right)\right. \\
& \left.+F\left(j_{1}+l_{1}, j_{2}, \ldots, j_{K}\right)+\lambda_{1}\right\}, \\
& \begin{array}{c}
\min _{1 \leq l_{2} \leq \min \left\{n_{2}-j_{2}+1, c\right\}}\left\{\theta_{l_{2}} l_{2} C\left(j_{1}-1, j_{2}+l_{2}-1, \ldots, j_{K}-1\right)\right. \\
\left.+F\left(j_{1}, j_{2}+l_{2}, \ldots, j_{K}\right)+\lambda_{2}\right\},
\end{array} \\
& \begin{array}{c}
\min _{1 \leq l_{2} \leq \min \left\{n_{2}-j_{2}+1, c\right\}}\left\{\theta_{l_{2}} l_{2} C\left(j_{1}-1, j_{2}+l_{2}-1, \ldots, j_{K}-1\right)\right. \\
\left.+F\left(j_{1}, j_{2}+l_{2}, \ldots, j_{K}\right)+\lambda_{2}\right\},
\end{array} \\
& , \ldots, \\
& \begin{array}{c}
\min _{1 \leq l_{K} \leq \min \left\{n_{K}-j_{K}+1, c\right\}}\left\{\theta_{l_{K}} l_{K} C\left(j_{1}-1, j_{2}-1, \ldots, j_{K}+l_{K}-1\right)\right. \\
\left.\left.+F\left(j_{1}, j_{2}, \ldots, j_{K}+l_{K}\right)+\lambda_{K}\right\}\right\}
\end{array} \\
& \begin{array}{c}
\min _{1 \leq l_{K} \leq \min \left\{n_{K}-j_{K}+1, c\right\}}\left\{\theta_{l_{K}} l_{K} C\left(j_{1}-1, j_{2}-1, \ldots, j_{K}+l_{K}-1\right)\right. \\
\left.\left.+F\left(j_{1}, j_{2}, \ldots, j_{K}+l_{K}\right)+\lambda_{K}\right\}\right\}
\end{array}
\end{aligned}
$$

The optimal value: $F(1,1, \ldots, 1)$.

Here, for any $j_{k}>n_{k}+1(k=1,2, \ldots, K)$,

$$
F\left(j_{1}, j_{2}, \ldots, j_{K}\right)=\infty \text {, }
$$

and

$$
\begin{aligned}
& C\left(j_{1}-1, j_{2}-1, \cdots, j_{i}+l_{i}-1, \cdots, j_{K}-1\right) \\
& =\left\{\begin{array}{cc}
\sum_{x=1, x \neq i}^{K} \sum_{k=0}^{j_{x}-1} p^{x}{ }_{k}+\sum_{k=0}^{j_{i}+l_{i}-1} p_{k}^{i}, & \text { if } \sum_{x=1, x \neq i}^{K} \sum_{k=0}^{j_{x}-1} p^{x}{ }_{k}+\sum_{k=0}^{j_{i}+l_{i}-1} p^{i}{ }_{k} \leq S ; \\
|I|+\sum_{x=1, x \neq i}^{K} \sum_{k=0}^{j_{x}-1} p^{x}{ }_{k}+\sum_{k=0}^{j_{i}+l_{i}-1} p_{k}^{i}, & \text { otherwise. }
\end{array}\right.
\end{aligned}
$$

in which $|I|$ is the time length of the unavailable interval.

The optimality of Algorithm DP can be demonstrated by the following theorem.

\section{Theorem}

The optimal schedule for the problem $(\mathrm{P})$ :

$$
1|r-a| V(\infty, c), \text { direct }|K| \Sigma \Sigma D_{j}^{i}+T
$$

can be obtained by Algorithm DP in $O\left(n^{K+2}\right)$ time.

\section{Proof}

By (2) of Lemma, there exists an optimal schedule in which jobs of each customer are processed in SPT order. We make jobs re-indexed to satisfy this fundamental condition. Moreover, the value of $F(1,1, \ldots, 1)$ is the minimum by choosing all possible values during all possible arrangements of $n$ jobs. In Algorithm DP, there are at most $n$ states because of $\sum_{i=1}^{K} n_{i}=n$. It takes no more than $O\left(n^{2}\right)$ time to calculate the value for each state. Therefore, the time complexity of Algorithm DP is bounded by $O\left(n^{K+2}\right)$.

\section{Instance}

To understand of our algorithm, consider an instance with three jobs, two customers, and a capacitated vehicle with capacity $\mathrm{c}=3$, where the first job and the second job will be delivered to customer 1 and the third job will be delivered to customer 2 . Thus, $n_{1}=2, n_{2}=1$. The processing times of the jobs are given in Table I. The beginning time and the end time of the unavailable interval on the machine is 4 and 6 , respectively, i.e., $[S, E]=[4,6]$. The cost of each delivery batch for the customer 1 is 2 and the cost of each delivery batch for the customer 2 is 4 , that is, $\lambda_{1}=2, \lambda_{2}=4$.

Now we need the completion times of all possible jobs' processing schedules (See Table II).

The performance of Algorithm DP is shown in the following.

Step 1. The initial condition:;

$$
F(3,2)=0 \text {; }
$$


TABLE II. COMPLETION TIMES OF All Schedules

\begin{tabular}{|c|c|c|c|c|}
\hline$C\left(j_{1}, j_{2}\right)$ & $j_{1}=0$ & $j_{1}=1$ & $j_{1}=2$ & $j_{1}=3$ \\
\hline$j_{2}=0$ & 0 & 1 & 3 & 3 \\
\hline$j_{2}=1$ & 2 & 3 & 7 & 7 \\
\hline$j_{2}=2$ & 2 & 3 & 7 & 7 \\
\hline
\end{tabular}

Step 2. 1

$$
\begin{gathered}
F(3,1)=\min \left\{\operatorname { m i n } \left\{C(3,0)+F(4,1)+\lambda_{1},\right.\right. \\
2 C(2,0)+F(5,1)+\lambda_{1}, \\
\left.3 C(1,0)+F(6,1)+\lambda_{1}\right\}, \\
\min \left\{C(2,1)+F(3,2)+\lambda_{2},\right. \\
\left.\left.2 C(2,2)+F(3,3)+\lambda_{2}\right\}\right\} \\
=\min \{\min \{\infty, \infty, \infty\}, \min \{7+0+4\}\}=11 ;
\end{gathered}
$$

Step 2. 2

$$
\begin{gathered}
F(2,2)=\min \left\{\operatorname { m i n } \left\{C(2,1)+F(3,2)+\lambda_{1},\right.\right. \\
\left.2 C(3,1)+F(4,2)+\lambda_{1}\right\}, \\
\min \left\{C(1,2)+F(2,3)+\lambda_{2},\right. \\
\left.\left.2 C(1,3)+F(2,4)+\lambda_{2}\right\}\right\} \\
=\min \{\min \{7+0+2, \infty\}, \min \{\infty, \infty\}\}=9 ;
\end{gathered}
$$

Step 2. 3

$$
\begin{gathered}
F(2,1)=\min \left\{\operatorname { m i n } \left\{C(2,0)+F(3,1)+\lambda_{1},\right.\right. \\
\left.2 C(3,0)+F(4,1)+\lambda_{1}\right\}, \\
\min \left\{C(1,1)+F(2,2)+\lambda_{2},\right. \\
\left.\left.2 C(1,2)+F(2,3)+\lambda_{2}\right\}\right\} \\
=\min \{\min \{3+11+2, \infty\}, \min \{3+9+4, \infty\}\}=16 ;
\end{gathered}
$$

Step 2. 4

$$
\begin{gathered}
F(1,2)=\min \left\{\operatorname { m i n } \left\{C(1,1)+F(2,2)+\lambda_{1},\right.\right. \\
\left.2 C(2,1)+F(3,2)+\lambda_{1}\right\}, \\
\min \left\{C(0,3)+F(1,3)+\lambda_{2}\right\} \\
=\min \{\min \{3+9+2,2 \times 7+2\}, \min \{\infty\}\}=14 ;
\end{gathered}
$$

Step 3 The optimal value:

$$
\begin{gathered}
F(1,1)=\min \left\{\operatorname { m i n } \left\{C(1,0)+F(2,1)+\lambda_{1},\right.\right. \\
\left.2 C(2,0)+F(3,1)+\lambda_{1}\right\} \\
\min \left\{C(0,1)+F(1,2)+\lambda_{2},\right. \\
\left.\left.2 C(0,2)+F(1,3)+\lambda_{2}\right\}\right\} \\
=\min \{\min \{1+16+2,2 \times 3+11+2\}, \min \{2+14+4, \infty\}\} \\
=\min \{19,19,20\}=19
\end{gathered}
$$

Therefore, in the optimal solution I, The vehicle will depart from the manufacturer at time 3 to visit customer 1 to deliver two jobs and then at time 7 to visit customer 2 to deliver only one job. In the optimal solution II, The vehicle will then depart from the manufacturer at time 1 and 3 to deliver only one job for customer 1 and again at time 7 to deliver only one job for customer 2 . The optimal objective function value is 19 .

\section{CONCLUSION}

This paper studies the manufacture and delivery procedure. In the manufacture stage, jobs need to be processed on a single machine. If the processing of the job is interrupted, the job can be resumed processing after the machine becomes available again. In the delivery stage, amounts of vehicles with fixed load capacities deliver completed jobs of the same customer in batches to that customer. We focus on minimizing the sum of total departure time and total delivery cost. A dynamic programming algorithm DP is presented and we prove that Algorithm DP is optimal with time complexity $O\left(n^{K+2}\right)$ and it is effective through the performance of an instance.

\section{ACKNOWLEDGEMENTS}

The project supported by Chinese National Natural Science Foundation (No. 11601316), the key discipline 'Applied Mathematics' of Shanghai Polytechnic University (No. XXKPY1604) and Science Training for Youth Teacher of Shanghai Polytechnic University (No. 201513).

\section{REFERENCES}

[1] Z.L. Chen, "Integrated production and outbound distribution scheduling: Review and extensions," Oper. Res. Hanover, vol. A58, pp. 130-148, 2010.

[2] Z.L. Chen and G. L. Vairaktarakis, "Integrated scheduling of production and distribution operations," Manage. Sci. Hanover, vol. A51, pp. 614$628,2005$.

[3] N. G. Hall and C. N. Potts, "The coordination of scheduling and batch deliveries," Ann. Oper. Res. Dordrecht, vol. A135, pp. 41-64, 2005.

[4] C. L. Li, G. Vairaktarakis and C. Y. Lee, "Machine scheduling with deliveries to multiple customer locations," Eur. J. of Oper. Res. Amsterdam, vol. A164, pp. 39-51, 2005.

[5] I. Averbakh and Z. Xue, "On-line supply chain scheduling problems with preemption,” Eur. J. of Oper. Res. Amsterdam, vol. A181, pp. 500504, 2007.

[6] I. Averbakh, "On-line integrated production distribution scheduling problems with capacitated deliveries," Eur. J. of Oper. Res. Amsterdam, vol. A200, pp. 377-384, 2010.

[7] C. Y. Lee, "Machine scheduling with an availability constraint," J. Global Optim. Dordrecht, vol. A9, pp. 395-416, 1996.

[8] Y. Ma, C. Chu and C. Zuo, "A survey of scheduling with deterministic machine availability constraints," Comput. Ind. Eng. Oxford, vol. A58, pp. 199-211, 2010.

[9] X. Wang and T. C. E. Cheng, "Machine scheduling with an availability constraint and job delivery coordination," Nav. Res. Log. Hoboken, vol. A54, pp. 11-20, 2007.

[10] J. Fan, X. Lu and P. Liu, "Integrated scheduling of production and delivery on a single machine with availability constraint," Theor. Comput. Sci. Amsterdam, vol. A562, pp. 581-589, 2015. 